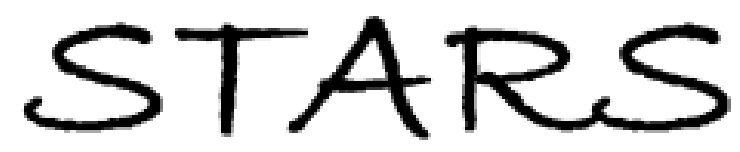

University of Central Florida

STARS

Faculty Bibliography 2000s

Faculty Bibliography

$1-1-2003$

\title{
Electron-inertia effects on driven magnetic field reconnection
}

\author{
N. Al-Salti \\ University of Central Florida \\ B. K. Shivamoggi \\ University of Central Florida
}

Find similar works at: https://stars.library.ucf.edu/facultybib2000

University of Central Florida Libraries http://library.ucf.edu

This Article is brought to you for free and open access by the Faculty Bibliography at STARS. It has been accepted for inclusion in Faculty Bibliography 2000s by an authorized administrator of STARS. For more information, please contactSTARS@ucf.edu.

\section{Recommended Citation}

Al-Salti, N. and Shivamoggi, B. K., "Electron-inertia effects on driven magnetic field reconnection" (2003). Faculty Bibliography 2000s. 3590.

https://stars.library.ucf.edu/facultybib2000/3590

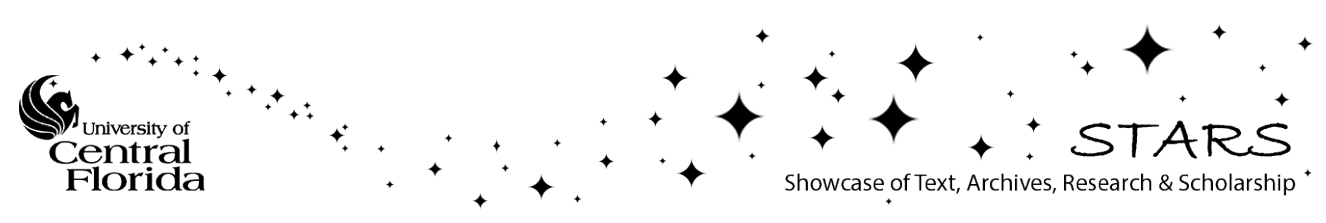




\section{Electron-inertia effects on driven magnetic field reconnection}

Cite as: Physics of Plasmas 10, 4271 (2003); https://doi.org/10.1063/1.1615242

Submitted: 26 March 2003. Accepted: 08 August 2003. Published Online: 17 October 2003

N. Al-Salti, and B. K. Shivamoggi

\section{ARTICLES YOU MAY BE INTERESTED IN}

The diffusion region in collisionless magnetic reconnection

Physics of Plasmas 6, 1781 (1999); https://doi.org/10.1063/1.873436

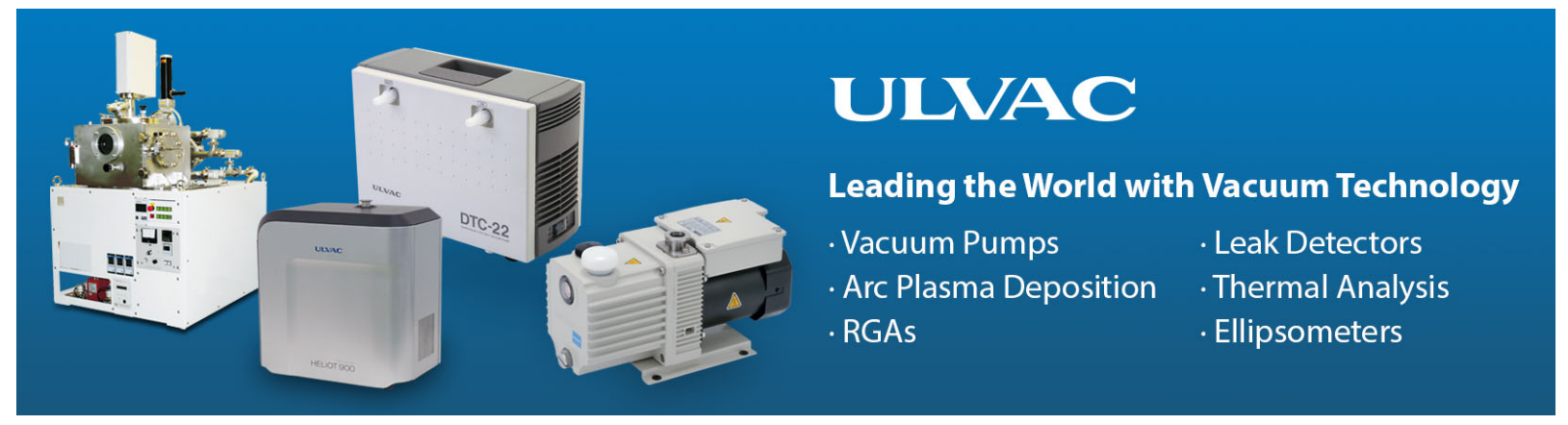




\title{
Electron-inertia effects on driven magnetic field reconnection
}

\author{
N. Al-Salti and B. K. Shivamoggi \\ University of Central Florida, Orlando, Florida 32816-1364
}

(Received 26 March 2003; accepted 8 August 2003)

\begin{abstract}
Electron-inertia effects on the magnetic field reconnection induced by perturbing the boundaries of a slab of plasma with a magnetic neutral surface inside are considered. Energetics of the tearing mode dynamics with electron inertia which controls the linearized collisionless magnetohydrodynamics (MHD) are considered with a view to clarify the role of the plasma pressure in this process. Cases with the boundaries perturbed at rates slow or fast compared with the hydromagnetic evolution rate are considered separately. When the boundaries are perturbed at a rate slow compared with the hydromagnetic evolution rate and fast compared with the resistive diffusion rate, the plasma response for early times is according to ideal MHD. A current sheet formation takes place at the magnetic neutral surface for large times in the ideal MHD stage and plasma becomes motionless. The subsequent evolution of the current sheet is found to be divided into two distinct stages: (i) the electron-inertia stage for small times (when the current sheet is very narrow); (ii) the resistive-diffusion stage for large times. The current sheet mainly undergoes exponential damping in the electron-inertia regime while the bulk of the diffusion happens in the resistivity regime. For large times of the resistive-diffusion stage when plasma flow is present, the current sheet completely disappears and the magnetic field reconnection takes place. When the boundaries are perturbed at a rate fast compared even with the hydromagnetic evolution rate, there is no time for the development of a current sheet and the magnetic field reconnection has been found not to take place. (C) 2003 American Institute of Physics. [DOI: 10.1063/1.1615242]
\end{abstract}

\section{INTRODUCTION}

The high-temperature plasmas in space (e.g., the corona and the magnetosphere where the plasma is of extremely low density so that the mean free path for binary particle collisions is several hundred times greater than the size of these systems) and in fusion systems have been found to be collisionless, so considerable work has gone into collisionless reconnection processes (Coppi et al., ${ }^{1-3}$ Schindler, ${ }^{4}$ Drake and Lee, ${ }^{5}$ Galeev, ${ }^{6}$ Wesson, ${ }^{7}$ Drake and Kleva, ${ }^{8,9}$ Ottaviani and Porcelli, ${ }^{10,11}$ and Shivamoggi, ${ }^{12,13}$ among others). Collisionless reconnection appears to be the origin of strong magnetic activity in solar flares $\left(\right.$ Shibata $\left.^{14}\right)$ and magnetospheric substorms (Nishida, ${ }^{15}$ Baker $^{16}$ ). The reconnection rate was found to increase by an order of magnitude in the electroninertia regime (Wesson, ${ }^{7}$ Ottaviani and Porcelli ${ }^{11}$ and Yamada et al. ${ }^{17}$ ). (Wesson ${ }^{7}$ suggests that the sudden appearance of the fast growth of the tokamak saw-tooth collapse might be related to the transition from the slow-resistive reconnection rate to the faster electron-inertial reconnection rate; the saw-tooth crash may occur on a time-scale small compared with the average electron-ion collision time, Edwards et $a l .{ }^{18}$ ) In the geophysical context, a collisionless reconnection regime in the magnetosphere implies much higher rates of solar wind entry than those indicated by the resistivitybased models.

Collisionless reconnection processes cannot be understood solely in terms of a single-fluid formulation of resistive magnetohydrodynamics (MHD). In a collisionless plasma, the electron-inertia leads to the decoupling of the plasma motion from that of the magnetic field lines, limits the elec- tron current and prevents it from becoming unbounded as the resistivity $\eta \Rightarrow 0$. In the electron-inertia regime, the conservation of the magnetic flux is replaced by the conservation of the generalized magnetic flux. This allows for the localized violation of the topological constraint on the magnetic flux and hence for reconnection to occur in the electron-inertia regime ${ }^{19}$ and an exchange between magnetic and kinetic energies. However, this process leads to the formation of current and vorticity layers of increasingly narrower microscales below the electron skin depth $d_{e}$ and then the resistive effects intervene inevitably.

One of the ways of inducing the magnetic field reconnection is to perturb the boundaries of a slab of plasma with a magnetic null surface inside (Fig. 1) - the Taylor problem (Kulsrud and $\mathrm{Hahm},{ }^{20,21} \mathrm{Hu},{ }^{22}$ Shivamoggi ${ }^{13,23,24}$ ). The boundaries were taken to be perfectly conducting walls. (The case with free plasma surface with constant pressure outside the plasma was not considered suitable to investigate the formation and disruption of current sheets in the interior of the plasma.) When the boundaries were perturbed at a rate slow compared with the hydromagnetic evolution rate but fast compared with the resistive diffusion rate, a current sheet develops at the magnetic neutral surface, and then disappears via resistive effects, causing the magnetic field reconnection to occur in the process. On the other hand, when the boundaries are perturbed at a rate fast compared even with the hydromagnetic evolution rate there is no time for the development of a current sheet and for the magnetic field reconnection to occur. The early treatments of this problem (Kulsrud and $\mathrm{Hahm},{ }^{20,21} \mathrm{Hu}^{22}$ ) were somewhat in disagreement which was partly due to the fact that these treatments 


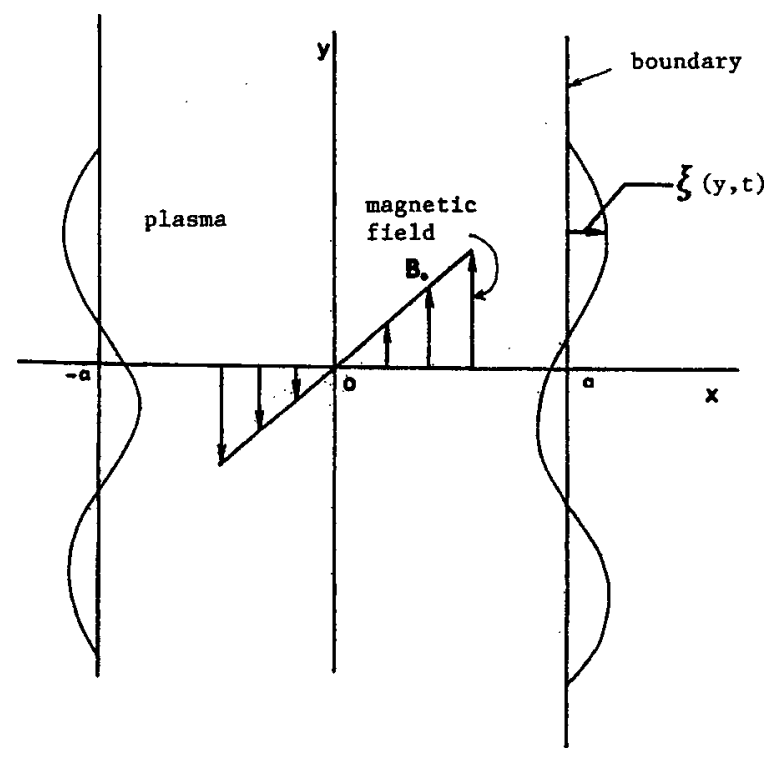

FIG. 1. Plasma slab with a magnetic neutral surface and perturbed boundaries.

had not looked at the adjustments occurring in the plasma flow associated with the reconnection process. These controversies were resolved by Shivamoggi, ${ }^{24}$ who made proper amends for the latter omission.

The electron-inertia effects on the Taylor problem were recently considered by Shivamoggi, ${ }^{13}$ who considered the case when the boundaries are perturbed at a rate fast compared with the resistive diffusion rate. The subsequent evolution of the current sheet formed at the magnetic neutral surface via ideal MHD development was found to be divided into two distinct stages: (i) the electron-inertia stage for small times (when the current sheet is very narrow); and (ii) the resistive-diffusion stage for large times.

The magnetic-field and plasma-flow profiles for these two stages were determined and matched with one another smoothly in the overlapping time interval; the ideal MHD solution was matched with that for the electron-inertia stage.

However, the solution describing the current sheet evolution discussed in Ref. 13 is based on the neglect of plasma flow which is valid for small times, but not for large times. In this paper, we consider the later phase when plasma flow is present $\left(\mathrm{Al}-\mathrm{Salti}^{25}\right)$. Another important question is whether the magnetic reconnection processes will occur as fast as required by the changing boundary conditions. Intuitively, it would appear that if the boundary conditions change too rapidly the magnetic reconnection processes would not have time to occur because they occur on the collisionless tearingmode time scale $\left(\mathrm{Al}-\mathrm{Salti}^{25}\right)$. This issue is investigated in this paper. Finally, since the plasma pressure does not show up explicitly in the equations governing collisionless MHD, it is not clear what role, if any, the plasma pressure plays in this collisionless reconnected process. We will therefore consider the energetics of the collisionless tearing mode, which controls the collisionless MHD, to clarify this issue, following the work of Adler et $a l .^{26}$ for the collisional tearing mode.

\section{STATEMENT OF THE MATHEMATICAL PROBLEM}

Consider a motionless plasma in a magnetic field of uniform gradient in the $x$ direction,

$$
\mathbf{B}=-B_{0} \frac{x}{a} \hat{\mathbf{i}}_{y}+B_{z} \hat{\mathbf{i}}_{z},
$$

and located between two boundaries at $x= \pm a$; see Fig. 1 .

Let us perturb the boundaries according to

$$
x= \pm(a+\xi),
$$

where

$$
\xi(y, t)=\alpha(t) \cos k y .
$$

Observe that the perturbations on the boundaries are out of phase with one another, since in-phase perturbations do not lead to magnetic field reconnection in the plasma (Kulsrud and $\mathrm{Hahm}^{20,21}$ ). Here $\alpha(t)$ is the time-dependent part of the boundary perturbation.

Let the magnetic field and velocity field consequent to this perturbation be given by

$$
\mathbf{B}=\boldsymbol{\nabla} \psi \times \hat{\mathbf{i}}_{z}+B_{z} \hat{\mathbf{i}}_{z}, \quad \mathbf{V}=\boldsymbol{\nabla} \phi \times \hat{\mathbf{i}}_{z} .
$$

We have assumed $B_{z}$ to be large (low- $\beta$ approximation) so the plasma flow may be taken to be incompressible.

We then obtain from the equations governing plasma flow and magnetic field transport (Shivamoggi ${ }^{13}$ ),

$$
\begin{aligned}
& \rho\left(\frac{\partial}{\partial t}+\mathbf{V} \cdot \boldsymbol{\nabla}\right) \nabla^{2} \phi=-\hat{\mathbf{i}}_{z} \cdot\left[\boldsymbol{\nabla} \psi \times \nabla\left(\nabla^{2} \psi\right)\right], \\
& \frac{\partial}{\partial t}\left(\psi-d_{e}^{2} \nabla^{2} \psi\right)+\left(\frac{\partial \phi}{\partial y} \frac{\partial}{\partial x}-\frac{\partial \phi}{\partial x} \frac{\partial}{\partial y}\right)\left(\psi-d_{e}^{2} \nabla^{2} \psi\right)=\hat{\eta} J,
\end{aligned}
$$

where $\rho$ is the mass density, $\hat{\eta} \equiv \eta c^{2}$ and $\eta$ is the resistivity of the plasma.

Now, let us write

$$
\begin{aligned}
& \psi(x, y, t)=\frac{B_{0}}{2 a} x^{2}+\psi_{1}(x, t) \cos k y, \\
& \phi(x, y, t)=\phi_{1}(x, t) \sin k y,
\end{aligned}
$$

and linearize about the magnetostatic equilibrium given by Eq. (1). We then obtain from Eqs. (5) and (6),

$$
\begin{aligned}
\frac{\partial}{\partial t}\left(\frac{\partial^{2} \phi_{1}}{\partial x^{2}}-k^{2} \phi_{1}\right) & =\frac{k B_{0} x}{a \rho}\left(\frac{\partial^{2} \psi_{1}}{\partial x^{2}}-k^{2} \psi_{1}\right), \\
\frac{\partial \psi_{1}}{\partial t}+\frac{k B_{0} x}{a} \phi_{1}= & \hat{\eta}\left(\frac{\partial^{2} \psi_{1}}{\partial x^{2}}-k^{2} \psi_{1}\right) \\
& +d_{e}^{2} \frac{\partial}{\partial t}\left(\frac{\partial^{2} \psi_{1}}{\partial x^{2}}-k^{2} \psi_{1}\right) .
\end{aligned}
$$

The boundary conditions,

$$
x= \pm(a+\xi): \quad \psi=\text { const }, \quad \frac{\partial \phi}{\partial y}= \pm\left(\frac{\partial \xi}{\partial t}-\frac{\partial \phi}{\partial x} \frac{\partial \xi}{\partial y}\right)
$$

which, on using Eq. (7), and linearizing, become 


$$
\begin{aligned}
& x= \pm a: \quad \psi_{1}=-B_{0} \alpha, \\
& k \phi_{1}= \pm \frac{d \alpha}{d t} .
\end{aligned}
$$

Observe that we have not imposed the no-slip condition on the plasma at the boundary which means that we are ignoring the presence of a boundary layer at $x= \pm a$ where viscous effects become important no matter how small the viscosity.

In order to determine the relative importance of the various terms in Eqs. (8) and (9), it is found to be useful to nondimensionalize them using the reference magnetic field $B_{0}$, channel width $a$ and a reference time scale $\tau$, as follows:

$$
\tilde{x} \equiv \frac{x}{a}, \quad \tilde{t} \equiv \frac{t}{\tau}, \quad \widetilde{\phi}_{1} \equiv k \tau B_{0} \phi_{1} .
$$

We then obtain

$$
\begin{gathered}
\frac{\tau_{\mathrm{A}}^{2}}{\tau^{2}} \frac{\partial}{\partial \tilde{t}}\left(\frac{\partial^{2} \widetilde{\phi}_{1}}{\partial \tilde{x}^{2}}-k^{2} a^{2} \widetilde{\phi}_{1}\right)=(k a) \tilde{x}\left(\frac{\partial^{2} \psi_{1}}{\partial \tilde{x}^{2}}-k^{2} a^{2} \psi_{1}\right), \\
\frac{\partial \psi_{1}}{\partial \tilde{t}}+(k a) \tilde{x} \phi_{1}= \\
\frac{\tau}{\tau_{R}}\left(\frac{\partial^{2} \psi_{1}}{\partial \tilde{x}^{2}}-k^{2} a^{2} \psi_{1}\right) \\
+\left(\frac{d_{e}^{2}}{a^{2}}\right) \frac{\partial}{\partial \tilde{t}}\left(\frac{\partial^{2} \psi_{1}}{\partial \tilde{x}^{2}}-k^{2} a^{2} \psi_{1}\right),
\end{gathered}
$$

where $\tau_{R} \equiv a^{2} / \eta$ is the resistive time scale, and $\tau_{\mathrm{A}}$ $\equiv a / B_{0} / \sqrt{\rho}$ is the Alvén-time scale.

Note that $d_{e}^{2} / a^{2} \ll 1$ so that the last bracketed term on the right-hand side in Eq. (13) is negligible except when the plasma dynamics develop narrow current and vorticity layers of the width of $O\left(d_{e}\right)$.

\section{ENERGETICS OF THE LINEARIZED COLLISIONLESS MHD}

In Eqs. (5) and (6), the pressure $p$ was eliminated, so it is not obvious what role, if any, the pressure $p$ plays in the linearized collisionless MHD which is controlled by the tearing mode dynamics. In order to investigate this aspect let us now discuss the energetics of the collisionless tearing mode with electron inertia. Energetics of the collisional tearing mode were considered by Adler et al. ${ }^{26}$

Let us now write

$$
\begin{aligned}
\psi(x, y, t)= & \psi_{0}(x)+\psi_{1}(x) e^{\gamma t} \cos k y \\
& +\left[\psi_{20}(x)+\psi_{22} \cos 2 k y\right] e^{2 \gamma t}, \\
\phi(x, y, t)= & \frac{\gamma}{k B_{0}} \phi_{1}(x) e^{\gamma t} \sin k y,
\end{aligned}
$$

with

$$
\psi_{0}^{\prime}=-B_{0} F .
$$

Linearizing in $\psi_{1}$ and $\phi_{1}$, Eqs. (5) and (6) give

$$
-\gamma^{2} \tau_{\mathrm{A}}^{2}\left(\phi_{1}^{\prime \prime}-k^{2} \phi_{1}\right)=F\left(\psi_{1}^{\prime \prime}-k^{2} \psi_{1}\right)-F^{\prime \prime} \psi_{1},
$$

$$
\psi_{1}-d_{e}^{2}\left(\psi_{1}^{\prime \prime}-k^{2} \psi_{1}\right)-\left(F-d_{e}^{2} F^{\prime \prime}\right) \phi_{1}=\frac{1}{\gamma \tau_{R}}\left(\psi_{1}^{\prime \prime}-k^{2} \psi_{1}\right),
$$

where primes denote differentiation with respect to $x$ and we have nondimensionalized distances using the reference length $a$.

Let us seek a growth rate which is fast compared with the resistive diffusion rate but slow compared with the hydromagnetic evolution rate, i.e.,

$$
\frac{1}{\gamma_{R}} \ll \gamma \ll \frac{1}{\tau_{\mathrm{A}}} \text {. }
$$

The magnetic energy associated with this mode on including electron inertia is

$$
M=\frac{1}{2} \int\left(|\mathbf{B}|^{2}+d_{e}^{2} \mathbf{J}^{2}\right) d x d y,
$$

and on averaging over $y$, we obtain

$$
\begin{aligned}
\langle\mathbf{M}\rangle= & \frac{e^{2 \gamma t}}{4} \int\left[\left(\psi_{1}^{\prime 2}+k^{2} \psi_{1}^{2}+4 \psi_{0}^{\prime} \psi_{20}^{\prime}\right)+d_{e}^{2}\left(\psi_{1}^{\prime \prime 2}\right.\right. \\
& \left.\left.+4 \psi_{0}^{\prime \prime} \psi_{1}-2 k^{2} \psi_{1}^{\prime \prime} \psi_{1}+k^{4} \psi_{1}^{2}\right)\right] d x .
\end{aligned}
$$

Differentiating Eq. (20) with respect to $t$ and using Eq. (15), we obtain

$$
\begin{aligned}
\left\langle\frac{d \mathbf{M}}{d t}\right\rangle= & \frac{\gamma e^{2 \gamma t}}{2} \int\left[-\psi_{1} \psi_{1}^{\prime \prime}+k^{2} \psi_{1}^{2}+d_{e}^{2}\left(\psi_{1}^{\prime \prime}-k^{2} \psi_{1}\right)^{2}\right. \\
& \left.+4 B_{0} F^{\prime} \psi_{20}-4 d_{e}^{2} B_{0} F^{\prime} \psi_{20}^{\prime \prime}\right] d x \\
& +\left.\frac{\gamma e^{2 \gamma t}}{2}\left(-4 B_{0} F \psi_{20}+\psi_{1} \psi_{1}^{\prime}\right)\right|_{\text {boundary }} .
\end{aligned}
$$

Now, using Eq. (14) in Eq. (6) and averaging over $y$, we obtain

$4 B_{0}\left(\psi_{20}-d_{e}^{2} \psi_{20}^{\prime \prime}\right)+\left(\phi_{1} \psi_{1}\right)^{\prime}-d_{e}^{2}\left[\left(\psi_{1}^{\prime \prime}-k^{2} \psi_{1}\right) \phi_{1}\right]^{\prime}$

$=\frac{2 B_{0}}{\gamma \tau_{R}} \psi_{20}^{\prime \prime}$.

The kinetic energy associated with this mode is

$$
\mathbf{K}=\frac{\rho}{2} \int|\mathbf{V}|^{2} d x d y
$$

and on averaging over $y$, we obtain

$$
\langle\mathbf{K}\rangle=\frac{e^{2 \gamma t}}{4} \gamma^{2} \tau_{\mathrm{A}}^{2} \int\left(\phi_{1}^{\prime 2}+k^{2} \phi_{1}^{2}\right) d x .
$$

Integrating the first term of the integrand in Eq. (24) by parts, and using Eq. (16), we obtain

$$
\langle\mathbf{K}\rangle=\frac{e^{2 \gamma t}}{4} \int F \phi_{1}\left(\psi_{1}^{\prime \prime}-k^{2} \psi_{1}-\frac{F^{\prime \prime}}{F} \psi_{1}\right) d x,
$$

where we have neglected the boundary term which is $O\left(\gamma^{2} \tau_{\mathrm{A}}^{2}\right)$ smaller than those that are kept. Differentiating Eq. (25) with respect to $t$, we obtain 


$$
\begin{aligned}
\left\langle\frac{d \mathbf{K}}{d t}\right\rangle= & \frac{\gamma e^{2 \gamma t}}{2} \int\left[F \phi_{1}\left(\psi_{1}^{\prime \prime}-k^{2} \psi_{1}\right)+F^{\prime}\left(\phi_{1} \psi_{1}\right)^{\prime}\right] d x \\
& -\left.\frac{\gamma e^{2 \gamma t}}{2}\left(F^{\prime} \phi_{1} \psi_{1}\right)\right|_{\text {boundary }} .
\end{aligned}
$$

From Eqs. (21) and (26), we obtain

$$
\begin{aligned}
\frac{d}{d t}\langle\mathbf{K}+\mathbf{M}\rangle= & \frac{\gamma e^{2 \gamma t}}{2} \int\left\{[ F \phi _ { 1 } - \psi _ { 1 } + d _ { e } ^ { 2 } ( \psi _ { 1 } ^ { \prime \prime } - k ^ { 2 } \psi _ { 1 } ) ] \left(\psi_{1}^{\prime \prime}\right.\right. \\
& \left.-k^{2} \psi_{1}\right)+\left[4 B_{0}\left(\psi_{20}-d_{e}^{2} \psi_{20}^{\prime \prime}\right)\right. \\
& \left.\left.+\left(\phi_{1} \psi_{1}\right)^{\prime}\right] F^{\prime}\right\} d x+\frac{\gamma e^{2 \gamma t}}{2}\left(-4 B_{0} F \psi_{20}\right. \\
& \left.+\psi_{1} \psi_{1}^{\prime}-F^{\prime} \phi_{1} \psi_{1}\right)\left.\right|_{\text {boundary }} .
\end{aligned}
$$

Using the result,

$$
\begin{aligned}
\int\left[\left(\psi_{1}^{\prime \prime}-k^{2} \psi_{1}\right) \phi_{1}\right]^{\prime} F^{\prime} d x= & \left.\left(\psi_{1}^{\prime \prime}-k^{2} \psi_{1}\right) \phi_{1} F^{\prime}\right|_{\text {boundary }} \\
& -\int F^{\prime \prime} \phi_{1}\left(\psi_{1}^{\prime \prime}-k^{2} \psi_{1}\right) d x,
\end{aligned}
$$

(27) then becomes

$$
\begin{aligned}
\frac{d}{d t}\langle\mathbf{K}+\mathbf{M}\rangle= & \frac{\gamma e^{2 \gamma t}}{2} \int\left[F \phi_{1}-\psi_{1}+d_{e}^{2}\left(\psi_{1}^{\prime \prime}-k^{2} \psi_{1}\right)\right. \\
& \left.-d_{e}^{2} F^{\prime \prime} \phi_{1}\right]\left(\psi_{1}^{\prime \prime}-k^{2} \psi_{1}\right) d x \\
& +\frac{\gamma e^{2 \gamma t}}{2} \int\left\{4 B_{0}\left(\psi_{20}-d_{e}^{2} \psi_{20}^{\prime \prime}\right)+\left(\phi_{1} \psi_{1}\right)^{\prime}\right. \\
& \left.-d_{e}^{2}\left[\left(\psi_{1}^{\prime \prime}-k^{2} \psi_{1}\right) \phi_{1}\right]^{\prime}\right\} F^{\prime} d x+\frac{\gamma e^{2 \gamma t}}{2} \\
& \times\left[-4 B_{0} F \psi_{20}+\psi_{1} \psi_{1}^{\prime}-F^{\prime} \phi_{1} \psi_{1}\right. \\
& \left.+d_{e}^{2}\left(\psi_{1}^{\prime \prime}-k^{2} \psi_{1}\right) \phi_{1} F^{\prime}\right]\left.\right|_{\text {boundary }}
\end{aligned}
$$

Using Eqs. (17) and (22), in the limit as $\gamma \tau_{R} \Rightarrow \infty$, we obtain

$$
\begin{aligned}
\frac{d}{d t}\langle\mathbf{K}+\mathbf{M}\rangle= & \frac{\gamma e^{2 \gamma t}}{2}\left[-4 B_{0} F \psi_{20}+\psi_{1} \psi_{1}^{\prime}-F^{\prime} \phi_{1} \psi_{1}\right. \\
& \left.+d_{e}^{2}\left(\psi_{1}^{\prime \prime}-k^{2} \psi_{1}\right) \phi_{1} F^{\prime}\right]\left.\right|_{\text {boundary }} .
\end{aligned}
$$

We will show that Eq. (30) is equal to the work done by the pressure at the boundaries plus the energy radiated through the current layer surface via the Poynting flux. The latter is given by

$$
\begin{aligned}
P_{x} & =\left(\mathbf{E}_{1} \times \mathbf{B}_{1}\right)_{x}+\left(\mathbf{E}_{2} \times \mathbf{B}_{0}\right)_{x} \\
& =-E_{1 z} B_{1 y}-E_{2 z} B_{0 y} \\
& =\left(-\gamma \psi_{1} \psi_{1}^{\prime} \cos ^{2} k y-2 \gamma \psi_{20} \psi_{0}^{\prime}\right) e^{2 \gamma t},
\end{aligned}
$$

and on averaging over $y$, and using Eq. (15), we get

$$
\left\langle P_{x}\right\rangle=\frac{\gamma e^{2 \gamma t}}{2}\left(-\psi_{1} \psi_{1}^{\prime}+4 B_{0} F \psi_{20}\right) .
$$

By including the electron inertia, the linearized electron momentum-balance condition may be written as

$$
\begin{aligned}
\frac{d p}{d x} & =\left[\mathbf{J}_{0} \times\left(\mathbf{B}-d_{e}^{2} \nabla^{2} \mathbf{B}\right)\right]_{x}+\left[\left(\mathbf{J}-d_{e}^{2} \nabla^{2} \mathbf{J}\right) \times \mathbf{B}_{0}\right]_{x} \\
& =-J_{0 z}\left(B_{y}-d_{e}^{2} \nabla^{2} B_{y}\right)-\left(J_{z}-d_{e}^{2} \nabla^{2} J_{z}\right) B_{0 y} \\
& =\left[-\psi_{0}^{\prime \prime} \psi_{1 e}^{\prime}-\left(\psi_{1 e}^{\prime \prime}-k^{2} \psi_{1 e}\right) \psi_{0}^{\prime}\right] e^{\gamma t} \cos k y,
\end{aligned}
$$

where $p$ is the electron pressure (the ions being assumed cold) and $\psi_{1 e} \equiv \psi_{1}-d_{e}^{2} \nabla^{2} \psi_{1}$. Using Eqs. (15) and (16), and dropping terms of $O\left(\gamma^{2} \tau_{\mathrm{A}}^{2}\right)$, we then have

$$
\begin{aligned}
\frac{d p}{d x} & =\left[F^{\prime} \psi_{1 e}^{\prime}+\left(\psi_{1 e}^{\prime \prime}-k^{2} \psi_{1 e}\right) F\right] B_{0} e^{\gamma t} \cos k y \\
& \approx\left(F^{\prime} \psi_{1 e}\right)^{\prime} B_{0} e^{\gamma t} \cos k y .
\end{aligned}
$$

Hence, the work done per unit time by the pressure force is given by

$$
p V_{x}=\gamma\left(F^{\prime} \psi_{1 e}\right) \phi_{1} e^{2 \gamma t} \cos ^{2} k y,
$$

and on averaging over $y$, we obtain

$$
\left\langle p V_{x}\right\rangle=\frac{\gamma e^{2 \gamma t}}{2}\left[\psi_{1}-d_{e}^{2}\left(\psi_{1}^{\prime \prime}-k^{2} \psi_{1}\right)\right] \phi_{1} F^{\prime} .
$$

From Eqs. (30), (31), and (33), it is obvious that

$$
\frac{d}{d t}\langle\mathbf{K}+\mathbf{M}\rangle=-\left\langle P_{x}\right\rangle-\left\langle p V_{x}\right\rangle,
$$

which establishes the energy balance of the tearing mode with electron inertia.

In the absence of electron-inertia effects, Eqs. (30) and (33) reduce to the ones obtained for the collisional case (Adler et $a l .{ }^{26}$ ).

\section{BOUNDARIES PERTURBED AT A RATE SLOW COMPARED WITH THE HYDROMAGNETIC EVOLUTION RATE AND FAST COMPARED WITH THE RESISTIVE DIFFUSION RATE}

\section{A. Ideal MHD stage}

For early times $\tau \sim O\left(\tau_{\mathrm{A}}\right)$ (so $\tau / \tau_{R} \ll 1$ ), assuming that the characteristic length scale of the plasma dynamics is of $O(a)$, the plasma response to the boundary perturbation is according to ideal MHD. Further, assuming in Eqs. (8) and (9) that $|\partial q / \partial x| \gg|k q|$, this response is then governed by the following equations:

$$
\begin{aligned}
& \rho \frac{\partial}{\partial t}\left(\frac{\partial^{2} \phi_{1}}{\partial x^{2}}\right)=\frac{k B_{0} x}{a} \frac{\partial^{2} \psi_{1}}{\partial x^{2}}, \\
& \frac{\partial \psi_{1}}{\partial t}+\frac{k B_{0} x}{a} \phi_{1}=0,
\end{aligned}
$$

the solution of which is given by (Kulsrud and Hahm ${ }^{19,20}$ )

$$
y=-A \int_{0}^{s} \frac{\sin u}{u} d u,
$$


where $A$ is an arbitrary constant and

$$
s \equiv \frac{k x t}{\tau_{\mathrm{A}}} .
$$

Solution (36) has the following asymptotic properties $\left(\right.$ Shivamoggi $\left.{ }^{24}\right)$ :

$$
\psi_{1} \approx\left\{\begin{array}{l}
-\frac{2 A k x^{2} t}{\tau_{\mathrm{A}}}, \text { for small } t, \\
\mp \frac{A \pi x}{2}, x \gtrless 0, \text { for large } t .
\end{array}\right.
$$

Using Eq. (35), we get

$$
\phi_{1} \approx\left\{\begin{array}{l}
\frac{A a x}{B_{0} \tau_{\mathrm{A}}}, \text { for small } t, \\
0, \text { for large } t .
\end{array}\right.
$$

Using (4), the magnetic and velocity fields are then given by

$$
\begin{aligned}
& B_{1 y} \approx\left\{\begin{array}{l}
\frac{2 A k x t}{\tau_{\mathrm{A}}}, \quad \text { for small } t, \\
\pm \frac{A \pi}{2}, \quad x \gtrless 0, \text { for large } t ;
\end{array}\right. \\
& V_{1 y} \approx\left\{\begin{array}{l}
-\frac{A a}{B_{0} \tau_{\mathrm{A}}}, \text { for small } t, \\
0, \text { for large } t .
\end{array}\right.
\end{aligned}
$$

Equation (39) shows that, for small $t, B_{1 y}$ is continuous at $x=0$, so there is no current sheet. This result is due to the fact that the Alvén-wave velocity for this model drops to zero at $x=0$ which prevents the linear disturbance from reaching the surface $x=0$ in a finite time. Consequently, the magnetic field configuration remains essentially unperturbed at $x=0$. However, for large $t, B_{1 y}$ has a jump at $x=0$, so there is now a current sheet. On the other hand, Eq. (40) shows that, for small $t$, there is a uniform flow of plasma in the channel which disappears and the plasma becomes motionless for large $t$ when a current sheet forms.

\section{B. Electron-inertia/resistive stage}

\section{Early phase when plasma is motionless}

The discussion at the end of Sec. IV A shows that the early part of the current-sheet evolution takes place in a motionless plasma and is governed by resistivity and electron inertia [which materializes if the current sheet is very narrow with width of $O\left(d_{e}\right)$ ] and is described by the following initial-value problem that results from Eq. (9) $\left(\right.$ Shivamoggi $\left.{ }^{13}\right)$ :

$$
\begin{aligned}
& \frac{\partial J_{z}}{\partial t}=\hat{\eta} \frac{\partial^{2} J_{z}}{\partial x^{2}}+d_{e}^{2} \frac{\partial}{\partial t}\left(\frac{\partial^{2} J_{z}}{\partial x^{2}}\right), \\
& t=0: \quad J_{z}=2 J_{0} \delta(x),
\end{aligned}
$$

where $J_{z}$ is the $z$-component of the current density,

$$
J_{z} \approx-\frac{\partial^{2} \psi_{1}}{\partial x^{2}} \text {. }
$$

A comparison of (42) with Eq. (39) immediately shows that

$$
A=\frac{2 J_{0}}{\pi}
$$

Applying the Fourier transform to the initial-value problem (41), (42), leads to

$$
\begin{aligned}
& \frac{d J_{k}}{d t}+\frac{\hat{\eta} k^{2}}{1+k^{2} d_{e}^{2}} J_{k}=0, \\
& t=0: J_{k}=\sqrt{\frac{2}{\pi}} J_{0},
\end{aligned}
$$

where $J_{k}(t)$ is the Fourier transform of $J_{z}(x, t)$ with respect to $x$, defined by

$$
J_{k}(t)=\frac{1}{\sqrt{2 \pi}} \int_{-\infty}^{\infty} e^{i k x} J_{z}(x, t) d x .
$$

The solution of (44) and (45) is readily found to be

$$
J_{k}(t)=\sqrt{\frac{2}{\pi}} J_{0} e^{-\hat{\eta} k^{2} t /\left(1+k^{2} d_{e}^{2}\right)} .
$$

For small $t$, electron-inertia effects dominate (Shivamoggi ${ }^{13}$ ) and (47) may be rewritten as

$$
J_{k}(t)=\sqrt{\frac{2}{\pi}} J_{0} e^{-\left(\hat{\eta} t / d_{e}^{2}\right) /\left[1-1 /\left(1+k^{2} d_{e}^{2}\right)\right]},
$$

and, hence, can be approximated as follows:

$$
J_{k}(t) \approx \sqrt{\frac{2}{\pi}} J_{0} e^{-\left(\hat{\eta} t / d_{e}^{2}\right)}\left[1+\frac{\hat{\eta} t / d_{e}^{2}}{1+k^{2} d_{e}^{2}}+\ldots\right] .
$$

Inverting the Fourier transform, we obtain

$$
\begin{aligned}
J_{z}(x, t) \approx & \sqrt{\frac{2}{\pi}} J_{0} e^{-\left(\hat{\eta} t / d_{e}^{2}\right)}\left[2 \delta\left(\frac{x}{d_{e}}\right)+\left(\frac{\hat{\eta} t}{d_{e}^{2}}\right) e^{-|x| / d_{e}}\right. \\
& +\ldots] .
\end{aligned}
$$

Equation (49) shows that the current is damped exponentially by the electron-inertia effects.

The magnetic flux in the electron-inertia phase is given by

$$
\psi_{1} \approx \mp J_{0} e^{-\left(\hat{\eta} t / d_{e}^{2}\right)}\left[x+\left(\frac{\hat{\eta} t}{d_{e}^{2}}\right) e^{-|x| / d_{e}}+\ldots\right], \quad x \gtrless 0 .
$$

We then have for the magnetic field

$$
B_{1_{y}} \approx \pm J_{0} e^{-\left(\hat{\eta} t / d_{e}^{2}\right)}\left[1-\left(\frac{\hat{\eta} t}{d_{e}^{2}}\right) e^{-|x| / d_{e}}+\ldots\right], \quad x \gtrless 0 .
$$

Observe that as $t \Rightarrow 0$, Eqs. (50) and (51) completely agree, as they should, with the large $t$ results of ideal MHD, namely, (37) and (39).

On the other hand, resistive effects dominate for large $t$, and (47) can be approximated as follows: 


$$
J_{k}(t) \approx \sqrt{\frac{2}{\pi}} J_{0} e^{-\hat{\eta} k^{2} t}\left[1+\left(\frac{\hat{\eta} t}{d_{e}^{2}}\right) k^{4} d_{e}^{4}+\ldots\right] .
$$

Inverting the Fourier transform, we obtain

$$
J_{z}(x, t) \approx \frac{J_{0}}{\sqrt{\pi \hat{\eta} t}}\left[1+\frac{3}{4}\left(\frac{d_{e}^{2}}{\hat{\eta} t}\right)+\ldots\right] e^{-x^{2} /(4 \hat{\eta} t)} .
$$

The corresponding magnetic flux is given by

$$
\begin{aligned}
\psi_{1} \approx & -J_{0}\left[1+\frac{3}{4}\left(\frac{d_{e}^{2}}{\hat{\eta} t}\right)+\ldots\right]\left[x \operatorname{erf}\left(\frac{x}{\sqrt{4 \hat{\eta} t}}\right)\right. \\
& \left.+\sqrt{\frac{4 \hat{\eta} t}{\pi}} e^{-x^{2} /(4 \hat{\eta} t)}\right],
\end{aligned}
$$

which has the following asymptotic behavior, in the resistive regime:

$\psi_{1} \approx\left\{\begin{array}{l}\mp J_{0}\left[1+\left(3 d_{e}^{2} / 4 \hat{\eta} t\right)+\ldots\right] x, \quad x \gtrless 0, \text { for small } t, \\ -J_{0}\left[1+\left(3 d_{e}^{2} / 4 \hat{\eta} t\right)+\ldots\right] \sqrt{\frac{4 \hat{\eta} t}{\pi}}\left(1-\frac{x^{2}}{2 \hat{\eta} t}\right), \\ \text { for large } t .\end{array}\right.$

Using Eq. (9), we then obtain

$\phi_{1} \approx\left\{\begin{array}{l}0, \text { for small } t, \\ \frac{a J_{0}}{k B_{0} x^{2}}\left[1+\left(3 d_{e}^{2} / 4 \hat{\eta} t\right)+\cdots\right] \sqrt{\frac{\hat{\eta}}{\pi t}}, \text { for large } t .\end{array}\right.$

The corresponding magnetic and velocity fields are then given by

$B_{1 y} \approx\left\{\begin{array}{l} \pm J_{0}\left[1+\left(3 d_{e}^{2} / 4 \hat{\eta} t\right)+\ldots\right], \quad x \gtrless 0, \text { for small } t, \\ \frac{J_{0}}{\sqrt{\hat{\eta} \pi t}}\left[1+\left(3 d_{e}^{2} / 4 \hat{\eta} t\right)+\cdots\right] x, \text { for large } t .\end{array}\right.$

$V_{1 y} \approx\left\{\begin{array}{l}0, \text { for small } t \\ \frac{a J_{0}}{k B_{0} x^{2}}\left[1+\left(3 d_{e}^{2} / 4 \hat{\eta} t\right)+\ldots\right] \sqrt{\frac{\hat{\eta}}{\pi t}}, \text { for large } t .\end{array}\right.$

In the absence of electron-inertia effects, Eqs. (55)-(58) reduce to the ones obtained for the collisional case $\left(\right.$ Shivamoggi $\left.{ }^{23}\right)$. Moreover, (57) shows that the current sheet has completely disappeared for large $t$, since $B_{1 y}$ is continuous at $x=0$ ! On the other hand, (58) shows that a plasma flow appears again for large $t$. So, the above results for large $t$ are not reliable because, as Kulsrud and $\mathrm{Hahm}^{19}$ pointed out for the collisional case, the neglect of plasma flow in Eq. (41) is no longer correct.

\section{Later phase when plasma flow is present}

One finds from Eqs. (8) and (9) that the later part of the resistive diffusion of the current sheet is governed by $\frac{\partial^{2} \psi_{1}}{\partial x^{2}}-k^{2} \psi_{1}=0$

$\frac{\partial \psi_{1}}{\partial t}+\frac{k B_{0} x}{a} \phi=0$.

Equation (59), in conjunction with the boundary condition (11a), lead to

$$
\psi_{1}=-B_{0} \alpha \frac{\cosh k x}{\cosh k a}
$$

Equation (60) then leads to

$$
\phi_{1}=\frac{d \alpha}{d t} \frac{a \cosh k x}{k x \cosh k a} .
$$

Thus, we have for the magnetic and velocity fields, for large $t$,

$$
\begin{aligned}
& B_{1 y}=B_{0} k \alpha \frac{\sinh k x}{\cosh k a}, \\
& V_{1 y}=-a \frac{d \alpha}{d t}\left(\frac{k x \sinh k x-\cosh k x}{x^{2} \cosh k a}\right) .
\end{aligned}
$$

Equations (61)-(64) are similar to the ones obtained for the collisional case (Shivamoggi ${ }^{24}$ ), because the electroninertia effects become unimportant for large $t$. Equation (63) shows that, for large $t, B_{1 y}$ is continuous at $x=0$, so there is no current sheet. However, $\psi_{1}$, according to (61), shows a different topology characterized by islands, indicating that the magnetic field reconnection has occurred. Equation (64) shows that during the process of magnetic reconnection, a plasma flow appears again, which varies rapidly near the magnetic neutral surface.

\section{BOUNDARIES PERTURBED AT A RATE FAST COMPARED WITH THE HYDROMAGNETIC EVOLUTION RATE}

One finds from Eqs. (8) and (9) that the plasma response for this case is then governed by

$$
\begin{aligned}
& \frac{\partial^{2} \phi_{1}}{\partial x^{2}}-k^{2} \phi_{1}=0, \\
& \frac{\partial}{\partial t}\left(d_{e}^{2} \frac{\partial^{2} \psi_{1}}{\partial x^{2}}-\left(d_{e}^{2} k^{2}+1\right) \psi_{1}\right)=\frac{k B_{0} x}{a} \phi_{1} .
\end{aligned}
$$

Equation (65), in conjunction with the boundary condition (11b), yields

$$
\phi_{1}=\frac{1}{k} \frac{d \alpha}{d t} \frac{\sinh k x}{\sinh k a} .
$$

Equation (66) in conjunction with the boundary condition (11a), then leads to 


$$
\begin{aligned}
\psi_{1}= & -\frac{B_{0} \alpha}{a \sinh k a}\left\{x \sinh k x+2 k d_{e}^{2}[\cosh k x\right. \\
& \left.\left.-\frac{\cosh k a}{\cosh \sqrt{d_{e}^{2} k^{2}+1}\left(a / d_{e}\right)} \cosh \sqrt{d_{e}^{2} k^{2}+1}\left(x / d_{e}\right)\right]\right\} .
\end{aligned}
$$

The corresponding magnetic and velocity fields are then given by

$$
\begin{aligned}
B_{1 y}= & \frac{B_{0} \alpha}{a \sinh k a}\left[k x \cosh k x+\left(1+2 k^{2} d_{e}^{2}\right) \sinh k x\right. \\
& \left.-\frac{2 k d_{e} \sqrt{d_{e}^{2} k^{2}+1} \cosh k a}{\cosh \sqrt{d_{e}^{2} k^{2}+1}\left(a / d_{e}\right)} \sinh \sqrt{d_{e}^{2} k^{2}+1}\left(x / d_{e}\right)\right],
\end{aligned}
$$

$$
V_{1 y}=-\frac{d \alpha}{d t} \frac{\cosh k x}{\sinh k a} .
$$

In the absence of electron-inertia effects, Eqs. (69) and (70) reduce to the ones obtained for the collisional case (Shivamoggi ${ }^{24}$ ). Equation (69) shows that $B_{1 y}$ is continuous at $x=0$, so there is no current sheet formation at $x=0$. Hence, a magnetic field reconnection does not take place, as in the collisional case $\left(\right.$ Shivamoggi $\left.^{24}\right)$. Further, (70) shows that the plasma flow now varies smoothly near the magnetic neutral surface implying the absence of vorticity concentration there.

\section{DISCUSSION}

In this paper, we have considered electron-inertia effects on the magnetic field reconnection induced by perturbing the boundaries of a slab of plasma with a magnetic neutral surface inside. We have investigated the energetics of the tearing-mode dynamics with the electron inertia which control the linearized collisionless MHD with a view to clarify the role of the plasma pressure in this reconnection process. When the boundaries are perturbed at a rate slow compared with the hydromagnetic evolution rate and fast compared with the resistive diffusion rate, the plasma response for early times is according to ideal MHD. A current sheet formation takes place at the magnetic neutral surface for large times in the ideal MHD stage and plasma becomes motionless. The subsequent evolution of the current sheet is found to be divided into two distinct stages: (i) the electron-inertia stage for small times (when the current sheet is very narrow); (ii) the resistive-diffusion stage for large times.
The current sheet mainly undergoes exponential damping in the electron-inertia regime while the bulk of the diffusion happens in the resistivity regime. For large times of the resistive-diffusion stage when plasma flow is present, the current sheet completely disappears and the magnetic field reconnection takes place.

When the boundaries are perturbed at a rate fast compared even with the hydromagnetic evolution rate, there is no time for the development of a current sheet and the magnetic field reconnection has been found not to take place.

\section{ACKNOWLEDGMENT}

The authors are grateful to the referee for his valuable remarks.

${ }^{1}$ B. Coppi, Phys. Lett. A 11, 226 (1964).

${ }^{2}$ B. Coppi, G. Laval, and R. Pellat, Phys. Rev. Lett. 16, 1207 (1966).

${ }^{3}$ B. Coppi, R. Galvao, R. Pellat, M. N. Rosenbluth, and P. H. Rutherford, Sov. J. Plasma Phys. 2, 533 (1977).

${ }^{4}$ K. Schindler, J. Geophys. Res. 79, 2803 (1974).

${ }^{5}$ J. F. Drake and Y. C. Lee, Phys. Fluids 20, 1341 (1977).

${ }^{6}$ A. A. Galeev, Phys. Fluids 21, 1353 (1978).

${ }^{7}$ J. Wesson, Nucl. Fusion 30, 2545 (1990).

${ }^{8}$ J. F. Drake and R. G. Kleva, Phys. Rev. Lett. 66, 1458 (1991).

${ }^{9}$ J. F. Drake, R. G. Kleva, and M. E. Mandt, Phys. Rev. Lett. 73, 1251 (1994).

${ }^{10}$ M. Ottaviani and F. Porcelli, Phys. Rev. Lett. 71, 3802 (1993).

${ }^{11}$ M. Ottaviani and F. Porcelli, Phys. Plasmas 2, 4104 (1995).

${ }^{12}$ B. K. Shivamoggi, J. Plasma Phys. 58, 329 (1997).

${ }^{13}$ B. K. Shivamoggi, Phys. Lett. A 292, 134 (2001).

${ }^{14}$ K. Shibata, Adv. Space Res. 17, 9 (1996).

${ }^{15}$ A. Nishida, Geomagnetic Diagnostics of the Magnetosphere (SpringerVerlag, New York, 1978).

${ }^{16}$ D. N. Baker, J. Geophys. Res. 101, 12975 (1996).

${ }^{17}$ M. Yamada, H. Ji, S. Hsu, T. Carter, R. M. Kulsrud, and F. Trintchouk, Phys. Plasmas 7, 1781 (2000).

${ }^{18}$ A. W. Edwards, D. J. Campbell, W. W. Engelhardt, H. V. Fahrbach, R. D. Gill, R. S. Granetz, S. Tsuji, B. J. D. Tubbing, A. Weller, J. Wesson, and D. Zasche, Phys. Rev. Lett. 57, 210 (1986).

${ }^{19}$ However, the possibility of magnetic reconnection in collisionless cases does raise some questions of principle [Taylor, as quoted in Ottaviani and Porcelli (Ref. 11)]: "Since the process is reversible one may argue that the reconnection in such a system is only a transient phenomenon and the magnetic field will eventually unconnect." However, the essential presence of even a few particle collisions will inhibit the latter process.

${ }^{20}$ R. M. Kulsrud and T. S. Hahm, Phys. Scr. T2/2, 525 (1982).

${ }^{21}$ R. M. Kulsrud and T. S. Hahm, Phys. Fluids 28, 2412 (1985).

${ }^{22}$ P. N. Hu, Phys. Fluids 26, 2234 (1983).

${ }^{23}$ B. K. Shivamoggi, Phys. Fluids 30, 1228 (1987).

${ }^{24}$ B. K. Shivamoggi, Phys. Scr. 47, 817 (1993).

${ }^{25} \mathrm{~N}$. Al-Salti, "Collisionless driven magnetic field reconnection," M.S. thesis, University of Central Florida, 2002.

${ }^{26}$ E. A. Adler, R. M. Kulsrud, and R. B. White, Phys. Fluids 23, 1375 (1980). 